\title{
Abelian Homegeneous Factorisations of Graphs
}

\author{
Huihong $\mathrm{Wu}^{*}$, Shuqu Qian \\ School of Sciences, Anshun University, Anshun Guizhou, 561000, China. \\ * Corresponding author. Tel.:+86 15908530159; email: hhwu19801024@sina.com \\ Manuscript submitted January 10, 2016; accepted June 8, 2016. \\ doi: 10.17706/ijapm.2016.6.3.112-119
}

\begin{abstract}
A homogeneous factorisation of a graph $\Gamma$ is a partition of its arc set such that there exist vertex transitive subgroups $M<G \leq \operatorname{Aut}(\Gamma)$ such that $M$ fixes each part setwise of the partition and Gpreserves the partition and transitive permuting the parts. In the present paper, we study homogeneous factorisation with $\mathrm{M}$ abelian. We give some interesting characterizations and constructions of such factorisations.
\end{abstract}

Key words: Cayley graph, homogeneous factorisations, abelian group.

\section{Introduction}

Let $\Gamma$ be a graph with vertex set $\mathrm{V} \Gamma$ and arc set $\mathrm{A} \Gamma$. If there exist a partition $P=\left\{P_{1}, P_{2}, \cdots, P_{k}\right\}$ of the arc set $\mathrm{A} \Gamma$ and two subgroups $M<G$ of $A u t \Gamma$, such that

1) $M$ is transitive on the vertex set $V \Gamma$ and fixes each Pi setwise;

2) $P$ is a G-invariant partition and the induced action GP of $G$ on $P$ is transitive, we call that $(\Gamma, P)$ is a $(M, G)$-homogeneous factorisation of index k.

If $M$ is regular on $\mathrm{V} \Gamma$ and $M \triangleleft G$, then we call the factorisation an M-Cayley homogeneous factorisation; if, in particular, $M$ is an abelian group, we occasionally simply call that $\Gamma$ has a homogeneous factorisation, M-Cayley homogeneous factorisation, M-abelian homogeneous factorisation and M-circulant homogeneous factorisation. The purpose of this paper is to character M-abelian homogeneous factorisation.

The finite graphs homogeneous factorisation is initiated and researched by famous Algebra graphs theorem experts Praeger, Guralinck, and Saxl [1]. In 2003, Lim and Stringer gave characterizations for homogeneous factorisations of complete digraph and Edge-transitive homogeneous factorisations of complete graphs [2], [3]. In 2004, Cuaresma studied homogeneous factorisations of Johnson graph [4]. In 2007, Giudici, Li, Potocnik, and Praeger accomplished homogeneous factorisations of complete multipartite graphs [5]. Fang, Li, and Wang characterized transitive 1-factorizations of arc-transitive graphs [6]. The general theory of homogeneous factorisation was studied in [1], [7]. A necessary and sufficient condition for complete graphs having circulant homogeneous factorisations has been given by Praeger and $\mathrm{Li}$, for complete graphs having $(\mathrm{M}, \mathrm{G})$ circulant homogeneous factorisations under the condition that $\mathrm{G} / \mathrm{M}$ is a cyclic group [8]. This paper give a research in the base of the main work above.

\section{Constructions}

Let $R$ be a group and $M=R: H<G=M: L$ with $L$ a subgroup of $\operatorname{Aut}(M)$. Let $\Gamma=\operatorname{Cay}(R, S)$ and $\Gamma_{i}=\operatorname{Cay}\left(R, S_{i}\right)$ for $i=1,2, \cdots, k$ are Cayley graphs of $M$. Finally let $P_{i}=A \Gamma_{i}, \quad \mathrm{P}=\left\{P_{1}, P_{2}, \cdots, P_{k}\right\}$ and $\Sigma=\left\{S_{1}, S_{2}, \cdots, S_{k}\right\}$. 
Lemma 2.1. Using notation defined above, $(\Gamma, \mathrm{P})$ is a $(M, G)$ homogeneous factorisation if and only if the following conditions hold:

1) $H$ fixes each $S_{i}$.

2) $S=\bigcup_{1 \leq i \leq k} S_{i}$ and setwise $S_{i} \cap S_{j}=\varnothing$ for all $i \neq j$.

3) $\Sigma$ is a $L$-invariant partition and the induced action $L^{\Sigma}$ of $L$ on $\Sigma$ is transitive. In particular, if we choose $M=R$, then $(\Gamma, \mathrm{P})$ is a M-Cayley homogeneous factorisation if and only if the above conditions 2 and 3 hold.

The following construction of M-Cayley homogeneous factorisation is given in [5].

Construction 2.2. Let $M$ be a group and let ${ }^{S}$ be a subset of $M \backslash\{1\}$ preserved by some subgroup $H \leq \operatorname{Aut}(M)$. Let $\Gamma=\operatorname{Cay}(M, S), O=\left\{O_{1}, O_{2}, \cdots, O_{r}\right\}$ be the set of H-orbits in $S$. For each $i \in\{1,2, \cdots, r\}$, choose $x_{i} \in O_{i}$. Suppose that $H$ has a proper subgroup $R$ containing $R$. For each $i \in\{1,2, \cdots, r\}$, let $B_{i} \leq O_{i}$ be the R-orbit of the element $x_{i}$, and let $S_{1}=B_{1} \cup B_{2} \cup \cdots \cup B_{r}$. Choose a set $\left\{h_{1}, h_{2}, \cdots, h_{k}\right\}$ of coset representations of $R$ in $H$ such that $h_{1}=1$. Define $S_{i}=S_{1}^{h_{i}}$, let $P_{i}$ be the arc set of the Cayley graph $\Gamma_{i}=\operatorname{Cay}\left(M, S_{i}\right), \quad \mathrm{P}=\left\{P_{1}, P_{2}, \cdots, P_{k}\right\}$ and $G=M: H$. Then $(\Gamma, \mathrm{P})$ is a M-Cayey homogeneous factorisation.

Lemma 2.3. Let $R$ be a group and $\Gamma=\operatorname{Cay}(R, S)$ be a Cayley graph. Let $H \triangleleft L$ be subgroups of $\operatorname{Aut}(R, S)$. Suppose $L$ is transitive on $S$ and $H$ is intransitive on $S$, say $\Sigma=\left\{S_{1}, S_{2}, \cdots S_{k}\right\}$ is the set of orbits of $H$ on $S$. Then there exists $(\Gamma, \mathrm{P})$ to be a $(M, G)$-homogeneous factorisation of index $k$, where $R \leq M \leq R: H$ and $G=R: L$.

Proof: Because $L$ is transitive on $S, H$ is intransitive on $S$, and $H \triangleleft L$. We have

$$
S=\alpha_{1}^{H} \bigcup \dot{\bigcup} \alpha_{2}^{H} \dot{\bigcup} \cdots \dot{U} \alpha_{k}^{H}=S_{1} \bigcup \dot{U} s_{2} \bigcup \dot{U} \cdots \dot{U} s_{k} .
$$

Therefore, $S_{i}=\alpha_{i}^{H} \quad(i=1,2, \cdots, k)$ is a block for $L$. Let $\Sigma=\left\{S_{i}^{l} \mid l \in L\right\}$, then $\Sigma$ is a $L$-invariant partition. As $\forall S_{i} \in \Sigma, l \in L$, we have $S_{i}^{l}=\left(\alpha_{i}^{H}\right)^{l}=\alpha_{i}^{l H}=\left(\alpha_{i}^{l}\right)^{H}=\alpha_{j}^{H}=S_{j}$ for some $j$. Further, $L$ preserves $\Sigma$ and $L$ is transitive on $\Sigma, \Sigma=\left\{S_{1}, S_{2}, \cdots, S_{k}\right\}$. Let $\Gamma=\operatorname{Cay}(R, S)$. We conclude $(\Gamma, \mathrm{P}, M, G)$ satisfies conditions (i)-(iii) of Lemma 2.1. So we be sure that $\Gamma$ has a factorisation whose factors are all of the same valency. Let $\Gamma_{i}=\operatorname{Cay}\left(R, S_{i}\right) \quad(i=1,2, \cdots, k), \quad P=\left\{A \Gamma_{1}, A \Gamma_{2}, \cdots, A \Gamma_{k}\right\}$, then $(\Gamma, \mathrm{P})$ is a $(\hat{R}: H, \hat{R}: L)$ homogeneous factorisation of index $k$.

Let $M$ be a group with order bigger than 2. For a nonidentity automorphism $\alpha$ of $M$, the following construction gives a way to construct a M-Cayley cyclic ${ }^{(M, G)}$ homogeneous factorisation ${ }^{(\Gamma, \mathrm{P})}$ with $G=M:\langle\alpha\rangle$.

Construction 2.4. Let $M$ and $\alpha$ are as above. Suppose $o(\alpha)=n \geq 2$. Then we may choose $l \geq 2$ such that $S:=\left\{x \in M: l \| x^{\langle\alpha\rangle} \mid\right\}$ has at least 2 elements. That is, $S$ is the union set of all $\langle\alpha\rangle$ orbits on $M$, say $O_{1}, O_{2}, \cdots, O_{k}$, whose length are multiple of $l$. Choose $x_{i} \in O_{i}$, and suppose $t_{i}=\left|O_{i}\right| / l$. Define $S_{1}=\bigcup_{1 \leq i \leq k}\left\{x_{i}, x_{i}^{\alpha^{l}}, \cdots, x_{i}^{\alpha^{\left(t_{i}-1\right) l}}\right\}$, and $S_{j}=S_{1}^{\alpha^{j-1}}$ for $j=2,3, \cdots, l$. Further let $\Gamma=\operatorname{Cay}(M, S)$, and for $i=1,2, \cdots, l$, let $P_{i}$ be the arc set of $\Gamma_{i}=\operatorname{Cay}\left(M, S_{i}\right), \mathrm{P}=\left\{P_{1}, P_{2}, \cdots, P_{l}\right\}$ and $G=M:\langle\alpha\rangle$.

Lemma 2.5. $(\Gamma, \mathrm{P})$ is a M-Cayley cyclic $(M, G)$ homogeneous factorisation. 
Proof: let $\Gamma=\operatorname{Cay}(M, S), \alpha \in \operatorname{Aut}(M), o(\alpha)=n \geq 2$.

Claim 1: $\forall x \in S$, we have $x^{\alpha^{i}} \in S$.

Proof: $x^{\alpha^{i}} \in S$. because $\alpha^{i} \in\langle\alpha\rangle$, so $\left|\left(x^{\alpha^{i}}\right)^{\langle\alpha\rangle}\right|=\left|x^{a^{i}\langle\alpha\rangle}\right|=\left|x^{\langle\alpha\rangle}\right|$, therefore $x^{\alpha^{i}} \in S$. According to Claim 1, we know $S$ is the union set of some $\left\langle\langle\alpha\rangle\right.$-orbits in $M$ and $\langle\alpha\rangle \leq \operatorname{Aut}(M, S)$, suppose $t_{i}=\left|O_{i}\right| / l$

Define $S_{1}=\bigcup_{1 \leq i \leq k}\left\{x_{i}, x_{i}^{\alpha^{l}}, \cdots, x_{i}^{\alpha^{\left(t_{i}-1\right) l}}\right\}$ and $S_{j}=S_{1}^{\alpha^{j-1}}$ for $j=2,3, \cdots, l$.

Suppose $\Sigma=\left\{S_{1}, S_{2}, \cdots, S_{l}\right\}$, obviously, we know that $\langle\alpha\rangle$ preserve $\Sigma$ and transitive on $\Sigma$. Let $\Gamma_{i}=\operatorname{Cay}\left(M, S_{i}\right), \quad \mathrm{P}=\left\{P_{1}, P_{2}, \cdots, P_{l}\right\}$ and $G=M:\langle\alpha\rangle$, that is $G / M \cong\langle\alpha\rangle$, we say that $(\Gamma, \mathrm{P})$ is a M-Cayley cyclic $(M, G)$ homogeneous factorisation. By construction 2.4, we have the following interesting proposition.

Proposition 2.6. For a given group $M$, there is a $(M, G)$ homogeneous factorisation $(\Gamma, \mathrm{P})$ for some $\Gamma, \mathrm{P}, G$ if and only if $M>2$.

Proof: The sufficiency of the proposition follows directly by Construction 2.4. Since $M$ is transitive on $V \Gamma$, it follows $|M|>2$. Further, if $|M|=2$, then $\Gamma=K_{2}$ obviously has no homogeneous factorisation, which proves the necessity of the proposition.

\section{Abelian Homogeneous Factorisation of Complete Graphs}

Let $F_{q}$ be a field of order $q$ with $q=p^{n}$ and $p$ prime. Let $G L(n, p)$ be the group of all invertible transformation a vector space of dimension n over field $F_{q}$, and let $V=F_{p}^{n} \backslash\{0\}$. Then $V$ can be viewed the set of non-zero vectors of n-dimension vector space over field $F_{q}$. It is known that $G L(n, k)$ has natural action on $V$, and containing a cyclic subgroup, say $\{\sigma\}$, which is regular on $V$. we call the cyclic group $\{\sigma\}$ a Singer subgroup of $G L(n, p)$. Then $\{\sigma\} \cong G L(1, q) \leq G L(n, p)$. Using the Singer subgroup, we may prove that complete graphs of prime power vertices have abelian homogeneous factorisations of certain index.

Lemma 3.1. Let $\Gamma=K_{p^{n}}$ be the complete graph. Then $\operatorname{Aut}(\Gamma)=S_{p^{n}}$. For each $k \mid\left(p^{n}-1\right)$ and $k \geq 2$, there exists a $(M, G)$ homogeneous factorisation $(\Gamma, \mathrm{P})$ of index $k$ with $M$ containing a regular cyclic subgroup on $V \Gamma$.

Proof: Let $R=Z_{p^{n}}$ be the additive group of finite field $F_{p^{n}}$. Then $\Gamma=\operatorname{Cay}(R, S)$ with $S=R \backslash\{0\}$ and $\operatorname{Aut}(M)=G L(n, p)$. Suppose $\{\sigma\}$ is a Singer subgroup of $G L(n, p)$. Since $\{\sigma\} \cong Z_{p^{n}-1}$ is cyclic and $k \mid\left(p^{n}-1\right)$, we may choose $L=\{\sigma\}$ such that $k \mid l$ and choose $H=\left\{\sigma^{l / k}\right\}$. Let $M=R: L$ and $G=R: H$, then $M \leq G \leq \operatorname{Aut}(\Gamma)$ and $|H: L|=k$. Since $\{\sigma\}$ is regular on $S$, it follows $L$ and $H$ are semi-regular on $S$, and $H$ has exactly $t$ orbits, say $O_{1}, O_{2}, \cdots, O_{t}$, where $t:=\left(p^{n}-1\right) /|H|$. Choose $x_{i} \in O_{i}$ and let $X=\left\{h_{1}, h_{2}, \cdots, h_{k}\right\}$ be a set of coset representatives of $L$ in $H$. Define $S_{1}=\bigcup_{1 \leq i \leq t}\left(x_{i}\right)^{L}$ and $S_{j}=S_{1}{ }^{h_{j}}$ for $2 \leq j \leq k$. Let $P_{i}$ be the arc set of $\Gamma_{i}=\operatorname{Cay}\left(M, S_{i}\right), \mathrm{P}=\left\{P_{1}, P_{2}, \cdots, P_{k}\right\}$. Then $\left(K_{p^{n}}, \mathrm{P}\right)$ is a $(M, G)$ homogeneous factorisation of the complete graph $K_{p^{n}}$, with $M$ containing a regular subgroup $R$ on $V \Gamma$. In Lemma 3.1, if $L=1$, then $M=R$ is regular on $V\left(K_{p^{n}}\right)$. That is, $\left(K_{p^{n}}, \mathrm{P}\right)$ is a R-abelian homogeneous factorisation of the complete graph $K_{p^{n}}$. However, the next lemma proves that the complete graph $K_{p^{n}}$ has 
a circulant homogeneous factorisation if and only if ${ }^{p}$ is odd.

Lemma 3.2. Let $\Gamma=K_{p^{d}}$ be the complete graph with ${ }^{p}$ a prime, and let $M$ be a cyclic group of order $n$ of order $p^{d}$ acting regular on $V \Gamma$. Then there exist a partition of $A \Gamma$ and $G \leq N_{\operatorname{Aut}(\Gamma)}(M)$ such that $(\Gamma, \mathrm{P})$ is a M-circulant homogeneous factorisation of index $k \geq 2$ if and only if $k \mid(p-1)$.

Proof: We first prove the necessity of the condition in the lemma. Suppose $G=M: H$ for some $H \leq A u t(\Gamma)$. Since $\operatorname{Val}(\Gamma)=p^{d}-1$, it follows $k \mid p^{d}-1$. Suppose $\mathrm{P}=\left\{P_{1}, P_{2}, \cdots, P_{k}\right\}, \Gamma_{i}=\operatorname{Cay}\left(M, S_{i}\right)$ with $P_{i}=A \Gamma_{i}$ and $G=M: H$ for some $H \leq \mathrm{Z}_{n}^{*}$. Since the induce action $G^{\mathrm{P}}$ of $G$ is transitive on $\mathrm{P}, H$ is transitive on $\left\{S_{1}, S_{2}, \cdots, S_{k}\right\}$, so $k$ divides the order of $H$, thus $k \mid p^{d-1}(p-1)$ as $\left|Z_{n}^{*}\right|=p^{d-1}(p-1)$. Note, $k \mid p^{d}-1$, it easily follows that $k \mid p-1$.

We now prove the sufficiency of the condition in the lemma. First, as $k \mid p-1, p$ is odd. Identifying $M$ with the additive group of the ring $\mathrm{Z}_{p^{d}}$, then $\operatorname{Aut}(M)=\mathrm{Z}_{n}^{*}=\langle r\rangle \cong \mathrm{Z}_{p^{d-1}(p-1)}$ acts on $M$ by multiplication, where $r$ is a primitive root of $p^{d}$. That is, $p^{d-1}(p-1)$ is the minimal positive solution of the equation $r^{x} \equiv 1\left(\bmod p^{d}\right)$. Let $S=M \backslash\{0\}$, and let $H=\operatorname{Aut}(M)$. Then $H$ has exactly $d$ orbits: $1^{H}, p^{H}, \cdots,\left(p^{d}-1\right)^{H}$, and their length equal $p^{d-1}(p-1), p^{d-2}(p-1), \cdots, p-1$, respectively. Let $X=\left\{1, p, \cdots, p^{d-1}\right\}$ be a set of the orbits representatives of $H$ acting on $S$ by multiplication. Obviously, the stabilizer $H_{p^{i}} \subseteq H_{p^{j}}$ for each $i<j$, so $\left\langle H_{x}: x \in X\right\rangle=H_{p^{d-1}}=\left\langle r^{p-1}\right\rangle \cong Z_{p^{d-1}}$ is a subgroup of $H$ with index $p-1$. Further, since $H$ is abelian and $k \mid p-1$, there exists a subgroup $R$ containing $\left\langle H_{x}: x \in X\right\rangle$ such that $|H: R|=k$. By Construction 2.2, $K_{p^{d}}$ has a circulant homogeneous factorisation of index $k$.

In lemma 3.1. We prove that complete graph of prime power vertices has abelian homogeneous factorisation. This leads to the following question.

Question 3.3. For $n \geq 2$ not a prime power, whether the complete graph $K_{n}$ has abelian homogeneous factorisation?

The following proposition give a sufficient condition for the existence of abelian homogeneous factorisation of the complete graphs with not prime power vertices, thus provides a partial answer of Question 3.3.

Proposition 3.4. Let $\Gamma=K_{n}$ be the complete graph and let $n=p_{1}^{d_{1}} p_{2}^{d_{2}} \cdots p_{r}^{d_{r}}$ be the prime power facterisation of $n$. If $\left(p_{1}^{d_{1}}-1, p_{2}^{d_{2}}-1, \cdots, p_{r}^{d_{r}}-1\right) \neq 1$, then there exists a $(M, G)$ abelian homogeneous factorisation $\left(K_{n}, \mathrm{P}\right)$.

Proof: Suppose $k \mid\left(p_{1}^{d_{1}}-1, p_{2}^{d_{2}}-1, \cdots, p_{r}^{d_{r}}-1\right)$ with $k \geq 2$. Write $l_{i}=p_{i}^{d_{i}-1} / k$. Let $M_{i}=\mathrm{Z}_{p_{i}}^{d_{i}} \quad \mathrm{Mi}=\mathrm{Zpdii}$ for $i=1,2, \cdots, r$, and let $M=M_{1} \times M_{2} \times \cdots \times M_{r}$. Then, $\operatorname{Aut}(M)=G L\left(d_{1}, p_{1}\right) \times G L\left(d_{2}, p_{2}\right) \times \cdots \times G L\left(d_{r}, p_{r}\right)$. Let $\langle\sigma\rangle$ be the Singer subgroup of $G L\left(d_{i}, p_{i}\right)$ for each ${ }^{i}$. Then $H:\left\langle\left(\sigma_{1}^{l_{1}}, \sigma_{2}^{l_{2}}, \cdots, \sigma_{r}^{l_{r}}\right)\right\rangle$ is a subgroup of Aut(M) with order $k$. Write $\sigma=\left(\sigma_{1}^{l_{1}}, \sigma_{2}^{l_{2}}, \cdots, \sigma_{r}^{l_{r}}\right)$. Since $\left\langle\sigma_{i}^{l_{i}}\right\rangle \cong Z_{k}$ is semi-regular on $M_{i} \backslash\{1\}$, it follows $H$ is semi-regular on $M \backslash\{1\}$, so $S:=\left\{x \in M \| x x^{\langle\sigma\rangle} \mid=o(\sigma)\right\}=M \backslash\{1\}$, thus by Construction 2.4, $\Gamma=\operatorname{Cay}(M, S)=K_{n} \Gamma$ has a M-abelian homogeneous factorisation of index $o(\sigma)=k$. 


\section{Abelian homogeneous factorization of some complete multipartite graphs}

Lemma 4.1. If $M$ is a group and $L \leq M$ a subgroup of index $s$ and order $t$, then $\operatorname{Cay}(M, M \backslash L)$ is isomorphic to the complete multipartite graph $K_{s[t]}$. Conversely, if $\Gamma=K_{S[t]}$ is a complete multipartite graph and $M$ a regular group of automorphisms of $\Gamma$, then there exists a subgroup $L_{\text {of }}$ order ${ }^{t}$ and index $s$ in $M$ such that $\Gamma$ is isomorphic to $\operatorname{Cay}(M, M \backslash L)$.

proof: See, for example,[9, $P$ roposition2.2]

In this section, we let $M=P \times Q, P \cong Z_{p}^{2} \mathrm{P} \sim \mathrm{p}$ and $Q \cong Z_{q}^{2} \quad(p, q$ are primes), then

$$
\operatorname{Aut}(M)=\operatorname{Aut}(P) \times \operatorname{Aut}(Q) \cong G L(2, p) \times G L(2, q) .
$$

In $G L(2, p)$, the singer cyclic group is $G L\left(1, p^{2}\right) \cong Z_{p^{2}}-1<G L(2, p)$, so there exists $\beta \in G L(2, p)$, such that $o(\beta)=p, p-1, p+1, p^{2}-1$, further $G L\left(1, p^{2}\right)$ is regular on $Z_{p}^{2} \backslash\{1\}$.

The same reason, in $G L\left(2, q\right.$, ) there exists $\beta^{\prime} \in G L(2, q)$ of order $o\left(\beta^{\prime}\right)=q, q-1, q+1, q^{2}-1 . G L\left(1, q^{2}\right)$ is semiregular on $Z_{q}^{2} \backslash\{1\}$.

Take $\alpha=(x, y) \in \operatorname{Aut}(M)$ where $x \in\langle\beta\rangle, y \in\left\langle\beta^{\prime}\right\rangle$ such that $o(\alpha)=o(x)=o(y) \neq 1$.

Construction 4.2. $o(\beta)=p, o\left(\beta^{\prime}\right)=q-1,(p, q-1) \neq 1$ Claim: $(1) \Gamma \cong K_{p q^{2}[p]}$ or $(2) \Gamma \cong K_{p q[p q]}$. proof:

Let $\alpha=(x, y), x \in\langle\beta\rangle, y \in\left\langle\beta^{\prime}\right\rangle, o(x)=o(y)=o(\alpha)=(p, q-1)=p$, because $\left\langle\beta^{\prime}\right\rangle$ acts on $Z_{p}^{2}$ semi-regular, let $\left(\left(a_{1}, a_{2}\right),(1,1)\right) \in M, \quad\left(a_{1}, a_{2}\right) \in \mathrm{Z}_{p}^{2}, \quad(1,1) \in \mathrm{Z}_{q}^{2}$ and it is an identity, so we find fixed points of $\langle\alpha\rangle$ acts on $M$. That is, find fixed points of $\langle x\rangle$ acts on $\mathrm{Z}_{p}^{2}$.

Let $\langle x\rangle=T=\left\{\left(\begin{array}{ll}1 & b \\ 0 & 1\end{array}\right) \mid b \in F_{p}\right\} \cong Z_{p}<G L(2, p), \quad\left(a_{1}, a_{2}\right) \in Z_{p}^{2}$, we have $\left(a_{1}, a_{2}\right)^{x_{i}}=\left(a_{1}, a_{2}\right)\left(\begin{array}{cc}1 & i b \\ 0 & 1\end{array}\right)=\left(a_{1}, i b a_{1}+a_{2}\right)$, $(1 \leq i \leq p)$, Let $\left(a_{1}, a_{2}\right)=\left(a_{1}, a_{2}+i b a_{1}\right)$, then $a_{2}=a_{2}+i b a_{1}$, and $i b$ is arbitrary, so $a_{1}=0$, and $\left(0, a_{2}\right)$ is the fixed-points of $\langle x\rangle$ acts on $\mathrm{Z}_{p}^{2}\left(a_{2} \in \mathrm{Z}_{p}\right)$. That is, there is $p$ fixed-points. These points form a cycle group $L$ based on relation of $\mathrm{Z}_{p}$, and $|L|=p,|M: L|=p q^{2},|S|=|M \backslash L|=p^{2} q^{2}-p=p\left(p q^{2}-1\right)$, by lemma 4.1, we conclude $\Gamma \cong K_{p q^{2}[p]}$.

The same reason, when $o(\beta)=p, o\left(\beta^{\prime}\right)=q+1$ or $q^{2}-1 ; \quad(p, q+1) \neq 1, \quad\left(p, q^{2}-1\right) \neq 1$, then $\Gamma \cong K_{p q^{2}[p]}$.

Obviously, $p \mid q-1$, let $\beta^{\prime} \in G L(2, q)_{u} \cong \mathrm{Z}_{q}: \mathrm{Z}_{q-1}$. Let $\alpha=(x, y), x \in\langle\beta\rangle, y \in\left\langle\beta^{\prime}\right\rangle, o(\alpha)=o(x)=o(y)=p$

$$
\begin{gathered}
\langle x\rangle=\langle\beta\rangle=\left\{\left\{\begin{array}{ll}
1 & b \\
0 & 1
\end{array}\right) \mid b \in F_{p}\right\} \cong \mathrm{Z}_{p}<G L(2, p) \\
\langle y\rangle=\left\langle\left(\begin{array}{cc}
1 & 0 \\
0 & \frac{q-1}{p}
\end{array}\right) \mid d \neq 0\right\rangle \cong \mathrm{Z}_{p}<\left\langle\beta^{\prime}\right\rangle=\left\{\left(\begin{array}{ll}
1 & 0 \\
0 & d
\end{array}\right) \mid d \in F_{q} \backslash\{0\}\right\} \cong \mathrm{Z}_{q-1}
\end{gathered}
$$

Suppose $\left(a_{1}, a_{2}\right) \in Z_{p}^{2}$, We have 


$$
\left(a_{1}, a_{2}\right)^{x_{i}}=\left(a_{1}, a_{2}\right)\left(\begin{array}{cc}
1 & i b \\
0 & 1
\end{array}\right)=\left(a_{1}, i b a_{1}+a_{2}\right),(1 \leq i \leq p)
$$

Let $\left(a_{1}, a_{2}\right)=\left(a_{1}, a_{2}+i b a_{1}\right)$, then $a_{2}=a_{2}+i b a_{1}$, and $i b$ is arbitrary. So $a_{1}=0$, and $\left(0, a_{2}\right)$ is the fixed-points of $\langle x\rangle$ acts on $\mathrm{Z}_{p}^{2}\left(a_{2} \in \mathrm{Z}_{p}\right)$. That is, there is $p$ fixed-points.

Let $\left(b_{1}, b_{2}\right) \in \mathrm{Z}_{q}^{2}$

We have

$$
\left(b_{1}, b_{2}\right)^{y_{i}}=\left(b_{1}, b_{2}\right)\left(\begin{array}{cc}
1 & 0 \\
& i^{i \frac{q-1}{p}}
\end{array}\right)=\left(b_{1}, b_{2} d^{i \frac{q-1}{p}}\right), \quad(1 \leq i \leq p)
$$

Let $\left(b_{1}, b_{2}\right)=\left(b_{1}, b_{2} d^{i \frac{q-1}{p}}\right)$. Then $b_{2}=b_{2} d^{i \frac{q-1}{p}}$ and $d^{i \frac{q-1}{p}}$ is arbitrary. So $b_{2}=0$, and $\left(b_{1}, 0\right)$ is the fixed-points of $\langle y\rangle$ acts on $\mathrm{Z}_{q}^{2}\left(b_{1} \in \mathrm{Z}_{q}\right)$. That is, there is $q$ fixed-points. Therefore, there are $p q$ fixed-points when $\langle\alpha\rangle$ acts on $Z_{p}^{2} \times Z_{q}^{2}$.

Let

$$
\left\langle a_{1}\right\rangle=\mathrm{Z}_{p},\left\langle b_{1}\right\rangle=\mathrm{Z}_{q}
$$

Then

$$
\left\langle\left(a_{1}, 0\right),\left(b_{1}, 0\right)\right\rangle \cong \mathrm{Z}_{p} \times \mathrm{Z}_{q}
$$

Obviously, this is an abelion group denoted by $L$ and $|L|=p q \quad, \quad|M: L|=p q$, $|S|=|M \backslash L|=p^{2} q^{2}-p q=p q(p q-1)$. By lemma 4.1, we can conclude $\Gamma \cong K_{p q[p q]}$.

The same reason, we can draw the following conclusion

(i): $o(\beta)=p+1, \quad p^{2}-1, \quad o\left(\beta^{\prime}\right)=q, \quad(p, q+1) \neq 1, \quad\left(p, q^{2}-1\right) \neq 1$, then $\Gamma \cong K_{p^{2} q[q]}$;

(ii): $o(\beta)=p-1, \quad o\left(\beta^{\prime}\right)=q, \quad(p-1, q) \neq 1$, then $\Gamma \cong K_{p^{2} q[q]}$ or $\Gamma \cong K_{p q[p q]}$

Theorem 4.3. Let $M=P \times Q=\mathrm{Z}_{p}^{n} \times \mathrm{Z}_{q}^{n}, \quad p$ and $q$ are prime, and $n$ is positive integer, then $\operatorname{Aut}(M) \cong G L(n, p) \times G L(n, q)$. Suppose $\beta \in G L(n, p)$, we have $o(\beta)=p, p-1, p^{n-1}+C_{n}^{1} p^{n-2}+C_{n}^{2} p^{n-3}+\cdots+1, p^{n}-1$. The same reason, when $\beta^{\prime} \in G L(n, q)$, then $o\left(\beta^{\prime}\right)=q, q-1, q^{n-1}+C_{n}^{1} q^{n-2}+C_{n}^{2} q^{n-3}+\cdots+1, q^{n}-1$, According to the method of construction 4.2, we can construct $\Gamma$, such that $\Gamma$ has homogeneous factorisation, we have these conclusion as the below:

(1)

(i): $o(\beta)=p-1, \quad o\left(\beta^{\prime}\right)=q-1, q^{n-1}+C_{n}^{1} q^{n-2}+C_{n}^{2} q^{n-3}+\cdots+1, q^{n}-1$, and the orders of $\beta$ and $\beta^{\prime}$ are not coprime, then $\Gamma \cong K_{p^{n} q^{n}}$; 
(ii): $o(\beta)=p^{n-1}+C_{n}^{1} p^{n-2}+C_{n}^{2} p^{n-3}+\cdots+1, o\left(\beta^{\prime}\right)=q-1, q^{n-1}+C_{n}^{1} q^{n-2}+C_{n}^{2} q^{n-3}+\cdots+1, q^{n}-1$, and the orders of $\beta$ and $\beta^{\prime}$ are not coprime, then $\Gamma \cong K_{p^{n} q^{n}}$.

$o(\beta)=p^{n}-1, \quad o\left(\beta^{\prime}\right)=q-1, q^{n-1}+C_{n}^{1} q^{n-2}+C_{n}^{2} q^{n-3}+\cdots+1, q^{n}-1$, and the orders of $\beta$ and $\beta^{\prime}$, are not coprime, then $\Gamma \cong K_{p^{n} q^{n}}$.

(2)

(i): $o(\beta)=p, \quad o\left(\beta^{\prime}\right)=q-1, \quad(p, q-1) \neq 1$, then $\Gamma \cong K_{p^{n-1} q^{n}[p]}$ or $\Gamma \cong K_{p^{n-1} q^{n-1}[p q]}$;

(ii): $o(\beta)=p-1, \quad o\left(\beta^{\prime}\right)=q, \quad(p-1, q) \neq 1$, then $\Gamma \cong K_{p^{n} q^{n-1}[q]}$ or $\left.\Gamma \cong K_{p^{n-1} q^{n-1}[p q]}\right]$;

(i): $o(\beta)=p, \quad o\left(\beta^{\prime}\right)=q^{n-1}+C_{n}^{1} q^{n-2}+C_{n}^{2} q^{n-3}+\cdots+1, q^{n}-1$, and the orders of $\beta$ and $\beta^{\prime}$, are not coprime, then $\Gamma \cong K_{p^{n-1} q^{n}[p]}$;

(ii): $o(\beta)=p^{n-1}+C_{n}^{1} p^{n-2}+C_{n}^{2} p^{n-3}+\cdots+1, p^{n}-1, \quad o\left(\beta^{\prime}\right)=q$, and the orders of $\beta$ and $\beta^{\prime}$ are not coprime, then $\Gamma \cong K_{p^{n} q^{n-1}[q]}$;

4.4. A method of construction connective subdigraph: In order to construct connective subdigraph, we need $M=\left\langle S_{1}\right\rangle$. Let

$$
S_{1}=\left\{\delta_{1}, \delta_{2}, \cdots, \delta_{m}\right\}, M \cong Z_{p}^{2} \times Z_{q}^{2}=\langle a, b\rangle \times\left\langle a^{\prime}, b^{\prime}\right\rangle
$$

We suppose

$$
\begin{gathered}
\delta_{1}=a \\
\delta_{i}=a^{\prime} b, i \neq 1 ; \\
\delta_{j}=b, \quad j \neq 1, i ;
\end{gathered}
$$

$\delta_{t} \in O_{t}, t \in 1,2, \cdots, m$ and $t \neq 1, i, j$; We have $\left\langle S_{1}\right\rangle=\left\langle a, a^{\prime}, b, b^{\prime}\right\rangle=M$, according to this method, we must be construct connective subdigraph.

In Theorem 4.3, we prove that the complete graph and the complete multipartite graph of product of two different primes power vertices has abeliant homogeneous factorisation. This leads to the following question.

Question 4.5: When

1) $\quad M=Z_{p}^{2} \times Z_{q}^{2} \times Z_{r}^{2}$ (v are primes);

2) $\quad M=\mathrm{Z}_{p_{1}}^{r_{1}} \times \mathrm{Z}_{p_{2}}^{r_{2}} \times \mathrm{Z}_{p_{3}}^{r_{3}}\left(p_{1}, p_{2}, p_{3}\right.$ are primes $)$;

Whether the complete graph and the complete multipartite graph have abeliant homogeneous factorization?

\section{Acknowledgment}

The authors acknowledge the support from the National Natural Science Foundation of China under Grants 61304146, the Provincial Science and Technology Foundation of Guizhou of China under Grant LKA201221 and 20152002, and the Provincial excellent creative talents of science and technology reward program of Guizhou of China under Grants 2014255. We would like to express our sincere appreciation on the anonymous reviewers for their insightful comments, which have greatly helped us in improving the 
quality of the paper.

\section{References}

[1] Guralnick, R., \& Li, C. H., \& Praeger, C. E., et al. (2004). On orbital partitions and exceptionality of primitive permutation groups, Trans. Amer. Math. Soc., 356, 4857-4872.

[2] Lim, T. K. (2003). Edge-transitive homogeneous factorisation of complete graphs. Ph.D. Thesis, the University of Western Australia.

[3] Stringer, L. (2004). Homogeneous factorisations of complete digraphs. M.Sc. Dissertation, the University of Western Australia.

[4] Cuaresma, M. C. N. (2004). Homogeneous factorisations of Johnson graphs. Ph. D. Thesis, University of the Philippines.

[5] Giudici, M., Li, C. H., Potocnik, P., \& Praeger, C. E. (2007). Homogeneous factorisation of complete multipartite graphs. Discrete Math, 307, 415-431.

[6] Fang X. G., Li, C. H., \& Wang, J. (2007). On transitive 1-factorisations of arc-transitive graphs. J. Combin. Theory Ser. A., 114, 692-703.

[7] Giudici, M., Li, C. H., Potocnik, P., \& Praeger, C. E. (2006). Homogeneous factorisation of graphs and digraphs. Europ. J. Combinatitics, 27, 11-37.

[8] Praeger, C. E., Li, C. H., \& Stringer, L. (2009). Common circulant homogeneous factorisations of the complete digraph. Discrete Math, 309, 3006-3012.

[9] Li, C. H. (2002). On isomorphisms of finite Cayley graphs -a survey. Discrete Math, 256, 301-334.

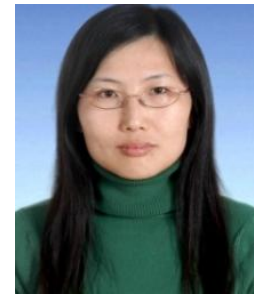

Huihong Wu received the B.S. degree in applied mathematics from Shanxi University, Shanxi, China, in 2001 and the M.S. degree in applied mathematics from Yunnan University, Yunnan, China, in 2009. She is currently an associate professor at School of Sciences, Anshun University. She has published over 20 journal papers, including computer application, computer engineering and so on.

Her research interests include groups and graph, evolutionary optimization and its application to Knapsack Problems, and mathematical modeling.

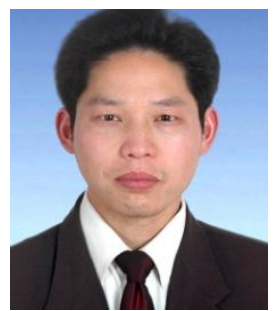

Shuqu Qian received the B.S. degree in mathematics and applied mathematics from FuYan Normal College, Anhui, China, in 2000 and the M.S. degree in operational research and cybernetics from the School of Guizhou University, Guizhou, China, in 2007. He is currently pursuing the Ph.D. degree in control theory and control engineering from the College of Automatic Engineering, Nanjing University of Aeronautics and Astronautics, Nanjing, China. Shuqu Qian is currently an associate professor at School of Sciences, Anshun University. He has published over 30 journal papers, including IEEE Transaction on Cybernetics, Control Theory and Application and so on.

His research interest includes immune optimization, evolutionary optimization and its application to power electronics, and mathematical modeling. 